\title{
Entransy Loss and its Application to Atkinson Cycle Performance Evaluation
}

\author{
Govind Maheshwari ${ }^{1}$, Sumer Singh Patel $^{2}$ \\ ${ }^{1,2}$ (Department of Mechanical Engineering, Institute of Engineering and Technology, Devi Ahilya University, \\ Indore, Madhya Pradesh 452017, India)
}

\begin{abstract}
Based on the concept of the entransy which characterizes heat transfer ability, a new Atkinson cycle performance evaluation criterion termed the entransy loss is established. Our analysis shows that the maximum entransy loss leads to the maximum output work, which is the maximum principle of entransy loss in thermodynamic processes. At the same time, it is found that minimum entropy generation alone could not describe change of the output work for the Atkinson cycle. The operation parameters are optimized for evaluating the maximum output work of Atkinson cycle by incorporating maximum entransy loss and minimum entropy generation when both, entransy loss and entropy generation, are induced by dumping the used streams into the environment is considered.
\end{abstract}

Keyword: Atkinson cycle; Environment; Entropy generation; Entransy loss; Maximum output work

\section{Introduction}

The Atkinson cycle is named after its inventor James Atkinson in 1882 [1]. As nearly $80 \%$ of the total energy consumption is related to heat, the optimization design of heat transfer and heat-work conversion has become one of important research topics under the increasing requirements on reducing energy consumption. The analyses of the heat transfer processes are very important for the optimization design of Atkinson cycle. In the last decades, some theories have been developed and applied to analyzing and optimizing these processes $[2,3]$. For heat transfer, it is irreversible in the view of thermodynamic, so entropy generation will produce during any heat transfer process. Bejan [3] applied the entropy generation minimization method to analyzing and optimizing heat transfer processes. The minimum principle of entropy generation is also an important criterion in process optimization. Because of its general features, the entropy concept has been extended to many other fields, such as thermodynamic optimization, quantum theory, and information theory by Shannon et al. [4] in 1948. These extended applications have already lead to great advancements, but doubts have arisen. For example, in the optimization of heating or cooling devices, minimum entropy generation does not always correspond to best performance [5,6]. For the minimum principle of entropy generation, it was noted that the heat exchanger effectiveness does not always increase with decreasing entropy generation when the concept of entropy generation is used to analyze heat exchangers [7]. Instead, it may become smaller under some conditions. Shah and Skiepko [8] also observed that the heat exchanger effectiveness can be maximum, intermediate or minimum at the maximum entropy generation in their discussion of 18 kinds of heat exchangers. Therefore, the minimum entropy generation principle is not always appropriate to heat transfer optimization.

In the last decade, Guo et al. [9] introduced a new physical parameter, entransy, which describes the heat transport ability. The new concept was proposed by the analogy between heat and electrical conductions [9]. Heat flow corresponds to electrical current, thermal resistance to electrical resistance, temperature to electric voltage, and heat capacity to capacitance. Entransy is actually the potential energy of the heat in a body, corresponding to the electrical energy in a capacitor. For an object whose internal energy is $E_{V}$ and temperature is $T$, its entransy is defined as [9]

$$
G=\frac{1}{2} E_{V} T,
$$

Entransy dissipation is always produced when heat is transfer from a high temperature body to low temperature by Chen et al. [10] in 2011. Guo et al. [9] derived the extremum entransy dissipation principle for heat transfer optimization. The maximum entransy dissipation corresponds to the maximum heat transfer rate for prescribed heat transfer temperature difference, while the minimum entransy dissipation corresponds to the minimum heat transfer temperature difference for prescribed the heat transfer rate. Furthermore, the extremum values of entransy dissipation always correspond to the minimum value of entransy -dissipation -based thermal resistance. Then, the extremum entransy dissipation principle is equivalent to the minimum thermal resistance principle. The principle of entransy theory are used to optimize heat conduction [11, 12], heat convection [13], thermal radiation $[14,15]$ and the optimization design of heat exchangers and heat exchangers network [16, 17], and are proved to be appropriate in heat transfer optimization. 
For thermodynamic cycles, the concept of entropy generation is used to describe the loss of the ability of doing work [18]. During a thermodynamic process, the more entropy generation is, the more the system would lose the ability of doing work by Ust et al. [19] in 2011. With this consideration, the concept of entropy generation has been widely used to optimize or analyze thermodynamic processes for performance improvement [20, 21]. Myat et al. [22] showed that the minimization of entropy generation in absorption cycle leads to the maximization of the COP when they analyzed an absorption chiller. However, there are also limitations on the entropy generation minimization in the optimization of thermodynamic cycles.

From above discussion, it could be found that the entransy theory is effective in heat transfer optimization, while the concept of entropy is not always.

The above introduction shows that there are limitation on application of entropy generation optimization of heat transfer processes and thermodynamic cycles. For the concept of entransy, it is appropriate in heat transfer optimization, but there are still different views when it is used to optimization thermodynamic cycles. On other hand, there are few reports on the applicability of entransy theory to the optimization design of thermodynamic cycles. Therefore, it is worth of making a further investigation on the optimization principles of thermodynamic cycles.

\section{Thermodynamic analysis of the Atkinson cycle}

The Atkinson cycle with irreversible processes is shown in Fig. 1, we consider the heat absorbed by the working medium $Q_{H}$ is not from the combustion reaction but from a high temperature stream through a counter flow heat exchanger whose number of heat transfer units is $N T U_{H}$, and $Q_{o}$ is the heat rate released to the environment by the used working medium and the output work is $W$. For the working fluid, the heat capacity flow rate at constant volume is $C_{V}$ and the heat capacity flow rate at constant pressure is $C_{P}$. The temperature of the working fluid is $T_{1}$ at state 1 and it gets increased to $T_{2}$ at state 2 by isentropic compression. The working fluid is heated by the high temperature stream from state 2 to 3 under constant volume and its temperature gets increased to $T_{3}$. The next process is the second isentropic process during which the mechanical work output is obtained and subsequently the temperature of the working fluid decreases to $T_{4}$ at state 4 . Finally, the working fluid is cooled by the low temperature fluid from state 4 to 1 under constant pressure and returns to its initial state.

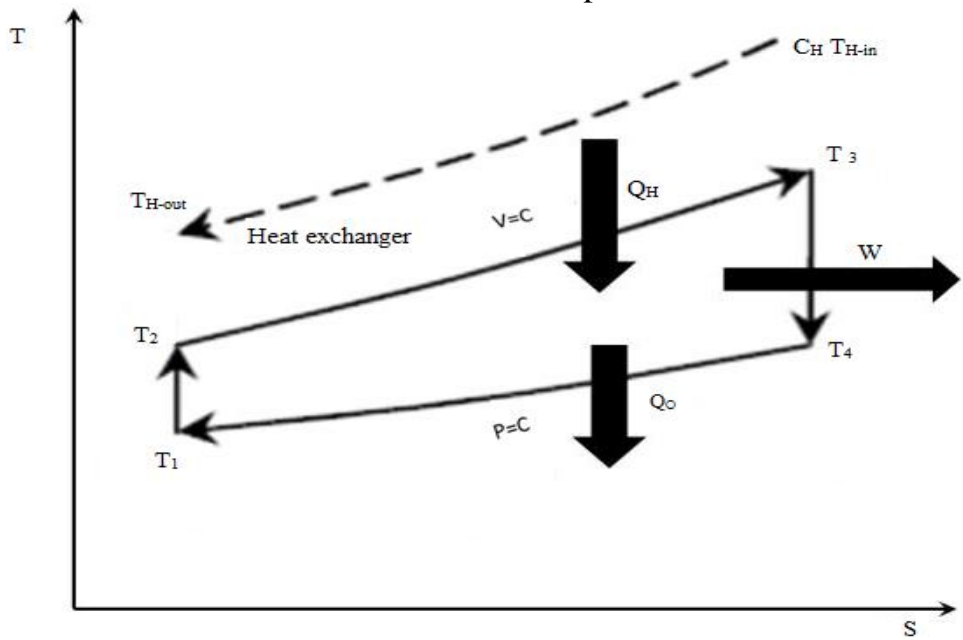

Figure 1: Sketch of the Atkinson cycle when the working medium is heated by a high temperature stream.

For the irreversible Atkinson cycle as shown in Fig. 1, the heat transfer rate $\left(\mathrm{Q}_{\mathrm{H}}\right)$ between the working fluid and the high temperature stream is given as [23].

$$
\begin{aligned}
Q_{H}=C_{H}\left(T_{H-\text { in }}-\right. & \left.T_{H-\text { out }}\right)=C_{H-\min }\left(T_{H-\text { in }}-T_{2}\right) \varepsilon_{H} \\
& =C_{H-\min }\left(T_{H-\text { in }}-T_{2}\right) \frac{1-\exp \left[-N T U_{H}\left(1-C^{H^{*}}\right)\right]}{1-C^{H^{*}} \exp \left[-N T U_{H}\left(1-C^{H^{*}}\right)\right]},
\end{aligned}
$$

Where, $C_{H-\min }$ is the smaller one between $C_{H}$ and $C_{V}, \varepsilon_{H}$ is the effectiveness, and $C^{H^{*}}$ is the capacity ratio of the high temperature stream. One can write the expression of $C^{H^{*}}$ as:

$C^{H^{*}}=\frac{\min \left(C_{H}, C_{V}\right)}{\max \left(C_{H}, C_{V}\right)}$, 
The heat rate $\left(\mathrm{Q}_{\mathrm{O}}\right)$ released to the environment by the used working medium is

$Q_{O}=C_{P}\left(T_{4}-T_{1}\right)$

Where $C_{P}$ is the heat capacity flow rate at constant pressure,

Based equation (2), one can get:

$T_{3}=T_{2}+\frac{Q_{H}}{C_{V}}$,

The output work (W)can be written as:

$W=Q_{H}-Q_{O}$

For the thermodynamic states 3 and 4, one can write:

$T_{3} v_{3}^{\gamma-1}=T_{4} v_{4}^{\gamma-1}$

Equation (9) can be rearranged as:

$T_{3}=T_{4} r_{e}^{\gamma-1}$,

Where, $r_{e}$ is the expansion ratio $=v_{4} / v_{3}$ and $\gamma$ is reversible adiabatic index $={ }^{C} / C_{V}$

Similarly for thermodynamic states 1 and 2, one can write:

$T_{1} v_{1}^{\gamma-1}=T_{2} v_{2}^{\gamma-1}$

Equation (10) can be rearranged as:

$T_{1}=\frac{T_{2}}{r_{k}^{\gamma-1}}$

Where, $r_{k}$ is the compression ratio $=v_{1} / v_{2}$

On combining equation (10) and (12), one can get:

$T_{3}=T_{2} * r_{e}^{\gamma} * \frac{1}{r_{k}^{\gamma}}$

And consequently,

$T_{4}=T_{1} * \frac{r_{e}}{r_{k}}$

2.1 Entropy generation when used stream is dumped into the Environment

The total entropy generation rate $\left(S_{g, e}\right)$ when used stream is dumped into the environment can be written as:

$S_{g, e}=C_{H} \ln \frac{T_{1}}{T_{H-i n}}+\frac{Q_{e}+Q_{O}}{T_{1}}$

Where $Q_{e}$ is the heat exchange between the used streams and the environment and $Q_{O}$ is the heat rate released to the environment by the used working medium: 
Where,

$Q_{e}=C_{H}\left(T_{H-o u t}-T_{1}\right)$

Using Equation (14) in equation (13), one can get:

$S_{g, e}=C_{H} \ln \frac{T_{1}}{T_{H-\text { in }}}+\frac{C_{H}\left(T_{H-o u t}-T_{1}\right)+Q_{O}}{T_{1}}$,

By using Esq. (2), one can get:

$T_{H-\text { out }}=T_{H-\text { in }}-\frac{Q_{H}}{C_{H}}$,

Using Equation (16) in Equation (15), one can get:

$S_{g, e}=C_{H} \ln \frac{T_{1}}{T_{H-i n}}+\frac{C_{H}\left(T_{H-i n}-\frac{Q_{H}}{C_{H}}-T_{1}\right)+Q_{O}}{T_{1}}$,

Equation (17) can be rearranged as:

$S_{g, e}=C_{H} \ln \frac{T_{1}}{T_{H-i n}}+\frac{C_{H}\left(T_{H-i n}-T_{1}\right)-\left(Q_{H}-Q_{O}\right)}{T_{1}}$,

Using Equation (6) in Equation (18), one can get:

$S_{g, e}=C_{H} \ln \frac{T_{1}}{T_{H-i n}}+\frac{C_{H}\left(T_{H-i n}-T_{1}\right)-W}{T_{1}}$,

\subsection{Entransy loss when used stream is dumped into the Environment}

Let us analyze the cycle from the entransy viewpoint. As the used high temperature stream and the used working medium are to be discharged into the environment, the entransy dissipation rate produced in the discharging process should be considered. The heat entransy flow rate from the high temperature stream is dissipated during the heat transfer process between the stream and the working medium and in the discharging processes of the used high temperature stream and the used working medium, some is used for doing work, and the rest is released into the environment. Therefore [24]

$G_{f-h}=G_{d i s}+G_{w-n e t}+G_{Q-e}$,

Where $G_{d i s}$ is the entransy dissipation, $G_{w-n e t}$ is the net work entransy flow rate of the cycle and $G_{Q-e}$ is the heat entransy flow that gets into the environment.

Where $G_{f-h}$ is the heat entransy flow rate from the high temperature stream, which is expressed as

$G_{f-h}=\frac{1}{2} C_{H}\left(T_{H-i n}^{2}-T_{1}^{2}\right)$,

$G_{Q-e}$ Is calculated by

$G_{Q-e}=\left(Q_{e}+Q_{o}\right) T_{1}$

It could be found that some entransy flow rate is lost in heat transfer, while some other is lost when the working medium does work. Therefore [24]

$G_{\text {loss }}=G_{d i s}+G_{w-n e t}=G_{f-h}-G_{Q-e}$, 
Using Equation (21) and (22) in equation (23), one can get:

$G_{\text {loss }}=\frac{1}{2} C_{H}\left(T_{H-i n}^{2}-T_{1}^{2}\right)-\left(Q_{e}+Q_{O}\right) T_{1}$,

Using Equation (14) in equation (24), one can get:

$G_{\text {loss }}=\frac{1}{2} C_{H}\left(T_{H-\text { in }}^{2}-T_{1}^{2}\right)-\left[C_{H}\left(T_{H-o u t}-T_{1}\right)+Q_{O}\right] T_{1}$,

Using Equation (16) in equation (25), one can get:

$G_{\text {loss }}=\frac{1}{2} C_{H}\left(T_{H-i n}^{2}-T_{1}^{2}\right)-\left[C_{H}\left(T_{H-i n}-\frac{Q_{H}}{C_{H}}-T_{1}\right)+Q_{O}\right] T_{1}$,

Equation (26) can be rearranged as:

$G_{\text {loss }}=\frac{1}{2} C_{H}\left(T_{H-i n}^{2}-T_{1}^{2}\right)-C_{H} T_{1}\left(T_{H-i n}-T_{1}\right)+\left(Q_{H}-Q_{O}\right) T_{1}$,

Using Equation (6) in Equation (27), one can get:

$G_{\text {loss }}=\frac{1}{2} C_{H}\left(T_{H-i n}^{2}-T_{1}^{2}\right)-C_{H} T_{1}\left(T_{H-i n}-T_{1}\right)+W T_{1}$,

\section{Results and discussion}

The derived expressions above are used and plotted in order to compare performance parameters of the Atkinson cycle with $T_{2}$. For the numerical calculations in the present work, the following values are used.

$C_{H}=2 \mathrm{~W} / \mathrm{K}, \quad c_{p}=1000 \mathrm{~J} /(\mathrm{kg} \mathrm{K}), \quad m=0.001 \mathrm{~kg} / \mathrm{s}, \quad \gamma=1.4, r_{k}=8, T_{H-i n}=420 \mathrm{~K}, T_{1}=300 \mathrm{~K}$, $N T U_{H}=2$.

Variations of the normalised output work, normalised entropy generation and normalised entransy loss with different values of $T_{2}$, when the entropy generation and the entransy loss induced by dumping the used stream into the environment is considered, are shown in Fig.2. As can be seen from the figure, values of normalised output work $\mathrm{W} / \max (\mathrm{W})$ goes on increasing with $\mathrm{T}_{2}$ and attains its maximum value at $\mathrm{T}_{2}=347 \mathrm{~K}$. On the other hand, values of normalised entropy generation $\mathrm{S}_{\mathrm{g}, \mathrm{e}} / \mathrm{max}\left(\mathrm{S}_{\mathrm{g}, \mathrm{e}}\right)$ goes on decreasing and normalised entransy loss $\mathrm{G}_{\text {loss }} / \mathrm{max}\left(\mathrm{G}_{\text {loss }}\right)$ goes on increasing with $\mathrm{T}_{2}$. The normalised entropy generation $\mathrm{S}_{\mathrm{g}, \mathrm{e}} / \max \left(\mathrm{S}_{\mathrm{g}, \mathrm{e}}\right)$ attains its minimum value at temperature $T_{2}=347 \mathrm{~K}$ while normalised entransy loss $\mathrm{G}_{\text {loss }} / \mathrm{max}\left(\mathrm{G}_{\text {loss }}\right)$ has its maximum value at $\mathrm{T}_{2}=347 \mathrm{~K}$. The above observations can also be drawn by evaluating Equations (6), (24) and (33).Hence, the minimum entropy generation and the maximum entransy loss both correspond to the maximum output work when dumping the used stream into the environment is considered. 


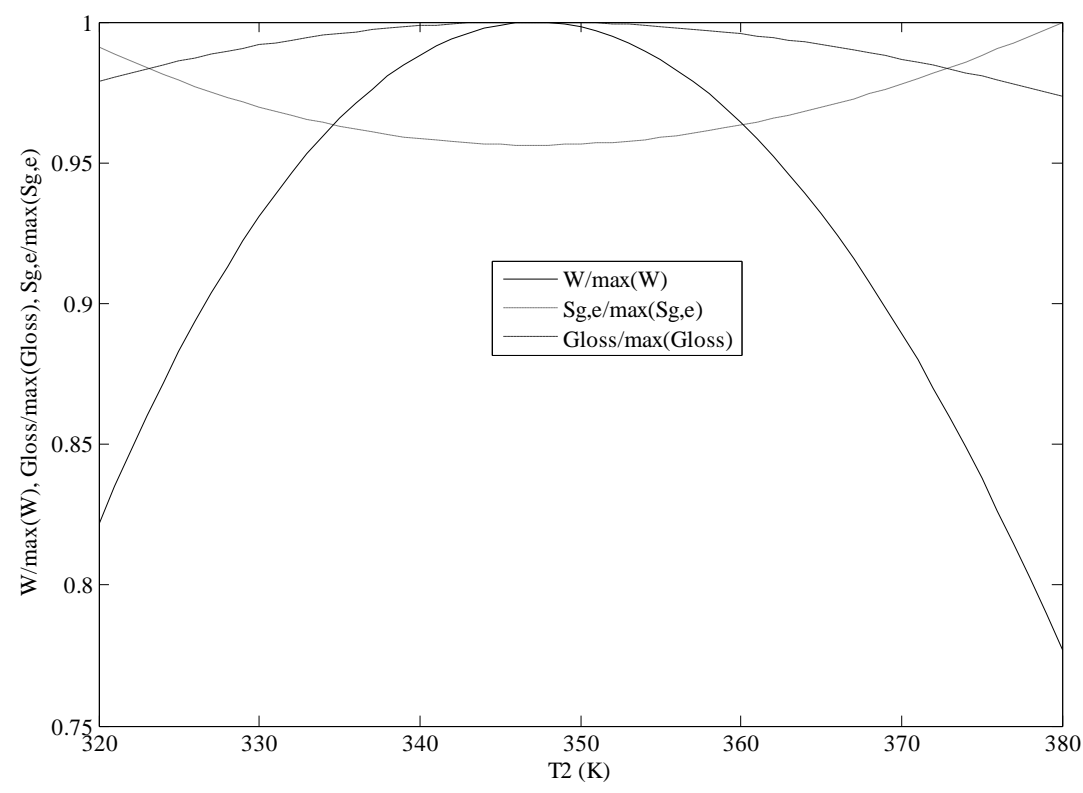

Fig. 2: Variations of the output work, the entropy generation and the entransy loss with $T_{2}$ when the entropy generation and the entransy loss induced by dumping the used stream into the environment are considered.

\section{Conclusion}

In this paper, the entransy and entropy optimization are applied to the Atkinson cycle. For any thermodynamic process, it is found that some of the net entransy flow from the heat sources is dissipated during the heating and the cooling processes of the working fluid, while the rest is lost in the process of doing work. The concept of entransy loss could be used to describe the performance of thermodynamic processes. With the maximum principle of entransy loss and the minimum principle of entropy generation, the thermodynamic optimization design of the Atkinson cycle are analyzed and discussed. It is found that the operation parameters could be optimized to get the maximum output work by calculating the maximum entransy loss and the minimum entropy generation when the entropy generation and the entransy loss induced by dumping the used stream into the environment are considered.

\section{Nomenclature}

$\begin{array}{ll}C & \text { heat capacity flow rate }\left(\mathrm{WK}^{-1}\right) \\ C_{P} & \text { heat capacity flow rate at constant pressure }\left(\mathrm{WK}^{-1}\right) \\ C_{V} & \text { heat capacity flow rate at constant volume }\left(\mathrm{WK}^{-1}\right) \\ G_{\text {loss }} & \text { entransy loss induced by environment }(\mathrm{WK}) \\ Q & \text { heat transfer rate }(\mathrm{W}) \\ S_{g, e} & \text { entropy generation induced by environment }\left(\mathrm{WK}^{-1}\right) \\ T & \text { temperature }(\mathrm{K}) \\ W & \text { output work }(\mathrm{W})\end{array}$

$\varepsilon \quad$ effectiveness of heat exchanger

$\gamma \quad$ reversible adiabatic index

\section{Subscripts}

$\begin{array}{cl}H & \text { hot fluid } \\ \text { in } & \text { inlet } \\ \max & \text { maximum } \\ \min & \text { minimum } \\ \text { out } & \text { outlet }\end{array}$




\section{References}

[1] Pulkrabek WW. Engineering Fundamentals of the Internal Combustion Engines, $2^{\text {nd }}$ Edn. Prentice-Hall, 2004

[2] Guo Z Y, Liu X B and Tao W Q, et al. Effectiveness- thermal resistance method for heat exchanger design and analysis, Int. J. Heat Mass Transfer, 53, 2010, 2877-2884.

[3] Bejan A, Advanced Engineering Thermodynamics ( $2^{\text {nd }}$ edn), Jhon Wiley \& Sons, New York, 1997.

[4] Shannon C E. A mathematical theory of communication. Bell Syst Tech J, 27, 1948, 379-423, 623-656.

[5] Liu X B, Meng J A and Guo Z Y. Entropy generation extremum and entransy dissipation extremum for heat exchanger optimization. Chinese Sci Bull, 54, 2009, 943-947.

[6] Liu X B and Guo Z Y. A novel method for heat exchanger analysis (in Chinese). Acta Phys Sin, 58, 2009, 4766- 4771.

[7] Bejan A. Second-law analysis in heat transfer and thermal design. Adv Heat Transfer, 15, 1982, 1-58.

[8] Shah R K and Skiepko T. Entropy generation extrema and their relationship with heat exchanger effectiveness- Number of transfer unit behaviour forComplex flow arrangements. J Heat Transfer, 126, 2004, 994-1002.

[9] Guo Z Y, Zhu H Y and Liang X G. Entransy - a physical quantity describing heat transfer ability. Int J Heat Mass Transfer, 50, 2007, 2545-2556.

[10] Chen L, Wei S and Sun F.Constructal entransy dissipation rate minimization of a disc, Int. J. Heat Mass Transfer, 54, 2011, 210216.

[11] Chen L, Wei S and Sun F. Constructal entransy dissipation rate minimization of round tube heat exchanger cross-section, Int. J. Them. Sci. 50, 2011, 1285- 1292

[12] Cheng X T, Xu X H and Liang X G. Homogenization of temperature field and temperature gradient field, Sci. China Ser. E-Tech. Sci. 52, 2009, 2937-2942.

[13] Yuan F and Chen Q. Two energy conservation principle in convective heat transfer optimization, Energy, 36, 2011, 5476-5485.

[14] Cheng X T and Liang X G. Entransy flux of thermal radiation and its application to enclosures with opaque surfaces, Int. J. Heat Mass Transfer, 54, 2011, 269-278.

[15] Cheng X T, Xu X H and Liang X G. Radiative entransy flux in enclosures with non isothermal or non-grey, opaque, diffuse surfaces and its application, Sci.

China: Tech. Sci. 54, 2011, 2446-2456.

[16] Cheng X T, Zhang Q Z and Liang X G. Analyses of entransy dissipation, entropy generation and entransy- dissipation -based thermal resistance on heat exchanger optimization, Appl. Therm. Eng. 38, 2012, 31-39.

[17] Cheng X T and Liang X G. Computation of effectiveness of two-stream heat exchanger network based on concept of entropy generation, entransy dissipation and entransy dissipation-based thermal resistance, Energy Convers.Manage. 58, 2012, 163-170.

[18] Zhao K H and Luo W Y. Thermotics (in Chinese), Higher Education Press, Beijing, 2002.

[19] Ust Y, Sahin B and Safa A. The Effects of Cycle Temperature and Cycle Pressure Ratios on the Performance of an Irreversible Otto Cycle, Acta Physica Polonica A, 120, 2011, 412-416.

[20] Adavbiele A S. Optimization of thermofluid system with second law, Int. J. Eng. Research Africa, 1, 2010, 67-80.

[21] Mistry K H, Lienhard J H and Zubair S M. Effect of entropy generation on the performance of humidification-ehumidification desalination cycle, Int. J. Them. Sci. 49, 2010, 1837-1874.

[22] Myat A, Thu K and Kim Y D. A second law analysis and entropy generation minimization of an absorption chiller. Appl Therm Eng, 31, 2011, 2405-2413.

[23] Yang S M and Tao W Q. Heat Transfer (in Chinese). Beijing: Higher Education Press, 1998

[24] Cheng XueTao, Wang WenHua and Liang XinGang. Entransy analysis of open thermodynamic systems, Engineering Thermophysics (in Chinese Science Bulletin), 57, 2012, 2934-2940. 